\title{
Prospectando rochas fluviais e artefatos do Paleolítico Médio Africano descobertos ao longo do Rio Cunene, fronteira entre Angola e Namíbia
}

\author{
Kathleen Nicoll \\ University of Utah, Department of Geography, Salt Lake City, Utah, U.S.A. Email: kathleen.nicoll@gmail.com
}

\begin{abstract}
Resumo:
Vestígios líticos do Paleolítico Médio Africano (PMA) preservados em corredores ribeirinhos como o perene Rio Cunene atesta a presença de atividades pré-históricas ao longo da atual divisa entre Angola e Namíbia. Próximo à Serra Cafema, mais de 30 vestígios líticos preservados em um contexto a céu aberto (plein-air) incluem lascas de quartzito, núcleos e pontas com alguma abrasão e polimento nos gumes, incluindo as primeiras pontas Levallois encontradas nesta região da África. Uma vez que a arqueologia desta região é pobremente conhecida, estas coleções culturais permitem correlações iniciais e fornecem uma base para reconstrução da proveniência, da procura e da produção de artefatos durante o PMA. Para avaliar as potenciais áreas-fonte de matéria-prima nesta região, métodos de contagem de seixos e avaliações composicionais foram conduzidos nos vestígios e no aluvião Quaternário (Qal) do terraço do rio. A fonte primária em potencial mais próxima de quartzito está em afloramentos rochosos localizados dentro de $2 \mathrm{~km}$ de distância do sítio, mas áreas-fonte confirmadas não foram identificadas especificamente. Uma hipótese emergente é que o Qal no terraço do Rio Cunene era uma fonte preferida para coleta de quartzito enquanto matéria prima pelos grupos móveis de caçadores-coletores durante o PMA após 225 mil anos atrás.
\end{abstract}

Palavras-chave: Paleolítico Médio Africano (PMA); quartzito, áreas de fonte lítica; aluvião Quaternário (Qal); Levallois; reconstrução da proveniência

\section{Introdução}

A reconstrução da produção e utilização de instrumentos de pedra - assim como da proveniência e propriedades das matérias primas - são imprescindíveis para a pesquisa arqueológica que fornece quadros teóricos para o comportamento pré-histórico humano (Ambrose \& Lorenz 1990; Kyara 1996). Estudos integrados do Paleolítico Médio Africano (PMA) - equivalente ao "African Middle Stone Age" (MSA) em inglês - formam nosso entendimento emergente das adaptações culturais que têm acompanhado a evolução biológica e comportamental e a expansão dos humanos modernos (e.g., Shultz et al. 2012; Wadley 2015). O período do PMA após 300 mil anos atrás engloba o desenvolvimento das tradições líticas regionais, a aparição de inovações tecnológicas importantes e a emergência e eflorescência da espécie Homo sapiens moderna (McBrearty \& Brooks 2000; Conard 2010;

Published by the School of History, Classics and Archaeology, University of Edinburgh ISSN: 2055-0472. URL: http://journals.ed.ac.uk/lithicstudies/

This work is licensed under a Creative Commons Attribution 2.5 UK: Scotland License. 
Dusseldorp 2011). O registro material do PMA preserva evidências de novos comportamentos simbólicos, incluindo inovações líticas e produção de instrumentos musicais, arte e joalheria com significado simbólico (Henshilwood et al. 2002; d'Errico 2003; d'Errico et al. 2003; Henshilwood \& Dubreuil 2009; Henshilwood \& d'Errico 2011; d'Errico \& Henshilwood 2011). Os registros destes humanos anatomicamente modernos fornecem hipóteses sobre suas habilidades cognitivas avançadas e estratégias para colonização de novos habitats e dispersão “fora da África” entre 80 mil anos e 60 mil anos atrás (Klein 1999; Henshilwood \& Marean 2003; 2006; Mellars 2006; Brown et al. 2012).

Uma vez que poucos sítios líticos do PMA têm sido registrados nas fronteiras modernas de Angola e Namíbia, nosso conhecimento de atividades humanas e padrões de assentamento nesta parte do sul da África é limitado (Mitchell 2002). Nicoll (2010) foi a primeira pesquisadora a publicar os achados pré-históricos desta área, que tem sido sujeita a poucas prospecções sistemáticas, e não há registros de escavações de sítios arqueológicos estratificados nas proximidades (Vogelsang 1998). No deserto hiperárido do noroeste da Namíbia, o pequeno tamanho das amostras líticas recuperadas impedem a definição de conjuntos de ferramentas, e os achados são de contextos geológicos que podem ter sido retrabalhados. Isto complica comparações com registros da África do Sul e além (e.g., Basell 2008; Deruiter et al. 2008; Jacobs et al. 2008; Porraz et al. 2013; Will et al. 2014; de la Peña 2015).

Apesar de suas limitações, os achados pré-históricos dispersos provenientes de contextos a céu aberto no sul da Angola e norte da Namíbia fornecem algumas bases para correlações de padrões taxonômicos artefatuais, e refletem atividades na paisagem em um espaço não circunscrito de caçadores-coletores móveis (Binford 1982; Oestmo et al. 2014). O sítio Cafema, do PMA, está localizado próximo à Serra Cafema $17^{\circ} 12^{\prime} 46^{\prime \prime}$ S, $12^{\circ} 12^{\prime} 6^{\prime \prime}$ L dentro de um corredor ribeirinho, no perímetro do hiperárido mar de areia Cunene, uma área ampla, plana e estéril coberta por depósitos arenosos de origem eólica (Figura 1). Artefatos foram descobertos no terraço do Rio Cunene, $\sim 16 \mathrm{~km}$ a oeste da área de Vale Hartmann e Marienfluss (Nicoll 2010).

O sítio Cafema é o primeiro em contexto estratigráfico direto com uma unidade de substrato seguramente datada; uma data de 220 mil anos atrás obtida por luminescência opticamente estimulada, mais especificamente por regeneração de alíquota única (OSL-SAR), fornece restrições iniciais de idade numa areia preservada dentro do terraço preenchido, e restringe uma idade máxima para a coleção arqueológica sobreposta (Nicoll 2010). Os mais de 30 achados líticos do PMA incluem lascas, núcleos, e pontas com graus de variação de abrasão e polimento dos gumes, assim como as primeiras pontas do tipo LevalloisMusteriense registradas nesta região da África entre 265 e 25 mil anos atrás (para uma revisão, veja Nicoll 2010, e referências). A produção de “pontas Levallois” (Brantingham \& Kuhn 2001) envolvem técnicas de lascamento que são especificamente desenvolvidas para preparar um núcleo para remoção de suportes com determinadas formas e contornos (Dibble \& Bar-Yosef 1995; Lycett \& Eren 2013). Evidências da preparação de núcleos usando a técnica Levallois clássica é relativamente incomum em coleções do PMA (Nicoll 2009; Lycett \& von Cramon-Taubadel 2013). 


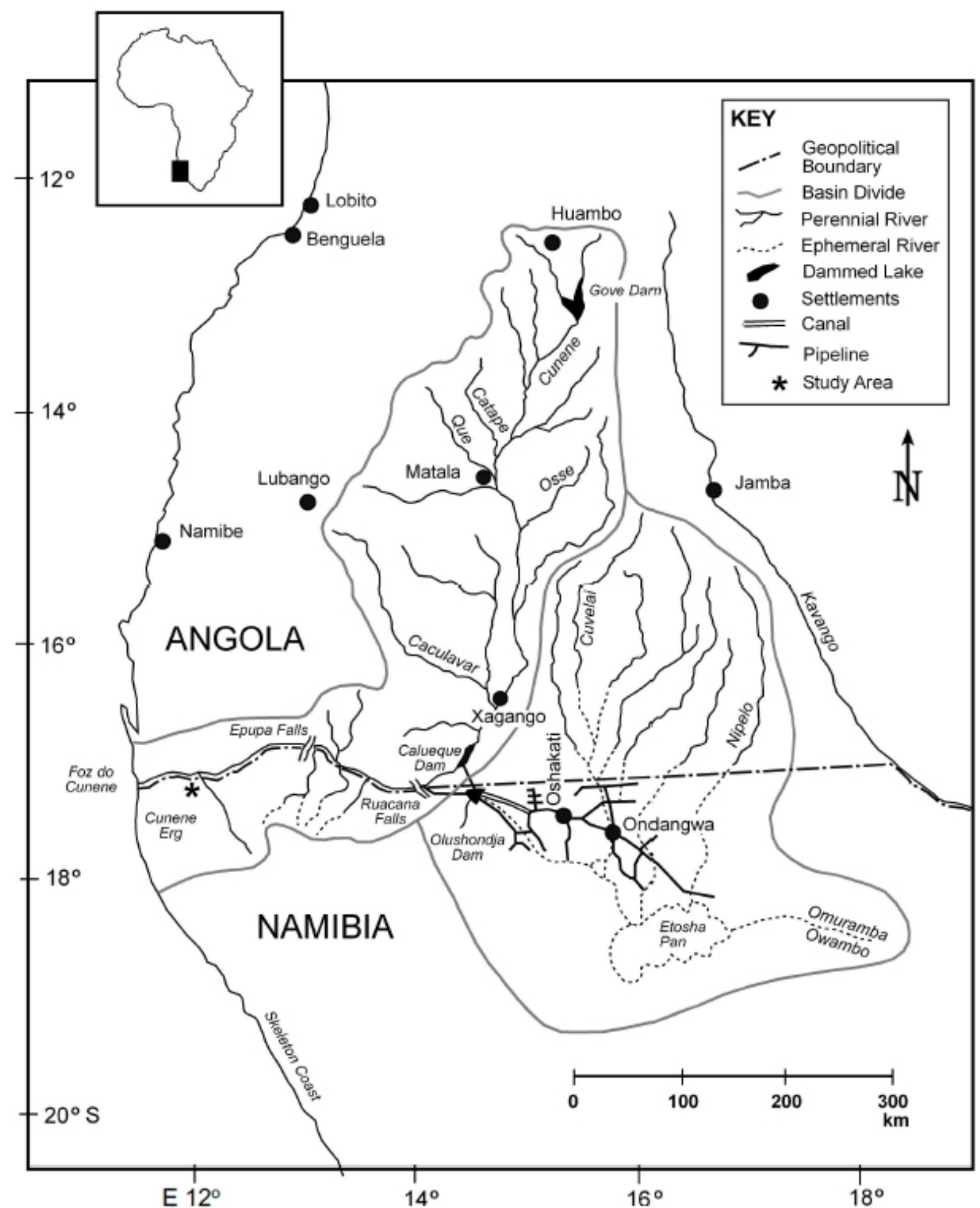

Figura 1. Mapa de localização do sul da África apresentando a bacia do Rio Cunene e a localização do sítio Cafema, indicado com uma estrela (Nicoll 2010). A bacia de drenagem (contornada) é subdividida em uma subbacia perene (ao norte) e uma sub-bacia efêmera, presentemente endorréica (ao sul). Assentamentos nas cascatas de maior energia incluem J-I-M (Jamba-Ia-Mina), Matunto, e Calueque.

Figure 1. Location map of southern Africa showing the watershed of the Cunene River and the location of Cafema site, denoted with a star just east of the Marienfluss (Nicoll 2010). The drainage basin (outlined) is subdivided into a perennial (northern) and an ephemeral, presently endorheic, sub-basin (southern). Settlements at the major stream knickpoints include J-I-M (Jamba-Ia-Mina), Matunto, and Calueque. 
Os achados provenientes de Cafema fornecem um contexto valioso para reconstrução da dispersão geográfica dos estilos líticos. Os artefatos de Cafema são relativamente rudimentares, e não se assemelham fortemente a outras entidades regionais e continentais (Nicoll 2010). Apesar da morfologia das lascas da coleção não serem diagnósticas, o número de lascas é significativo, e os artefatos não parecem terem sido retrabalhados. A determinação das áreas-fonte líticas locais pode fornecer modelos geográficos ligando inovações tecnológicas com movimentos regionais, dispersões residenciais e padrões de atividades nos corredores fluviais (Beck \& Jones 1990; Andrefsky 1994; 1995 e referências citadas). Tais modelos fornecem inferências sobre atividades oportunistas, sazonalidade, preferências de sítios específicos, e comportamentos de economia em distâncias crescentes em relação às áreas-fonte (ver: Renfrew 1972: 465; Renfrew et al. 1969: 327-329; Newman 1994; Rogers 1996, para exemplos).

Este artigo apresenta uma avaliação de proveniência dos artefatos líticos do PMA descobertos próximos a Cafema, na fronteira entre Angola e Namíbia (Nicoll 2010). A geologia regional e configuração geométrica foram estudados para relacionar a localização do sítio do artefato dentro de um terraço do Rio Cunene (Figura 2), às fontes locais em potencial que ocorrem enquanto afloramentos rochosos geológicos dentro da bacia de drenagem. Foram avaliadas a proveniência das matérias-primas através de mapeamento primário dos afloramentos geológicos na área, e descrito o aluvião Quaternário (Qal) do terraço do rio no sítio.

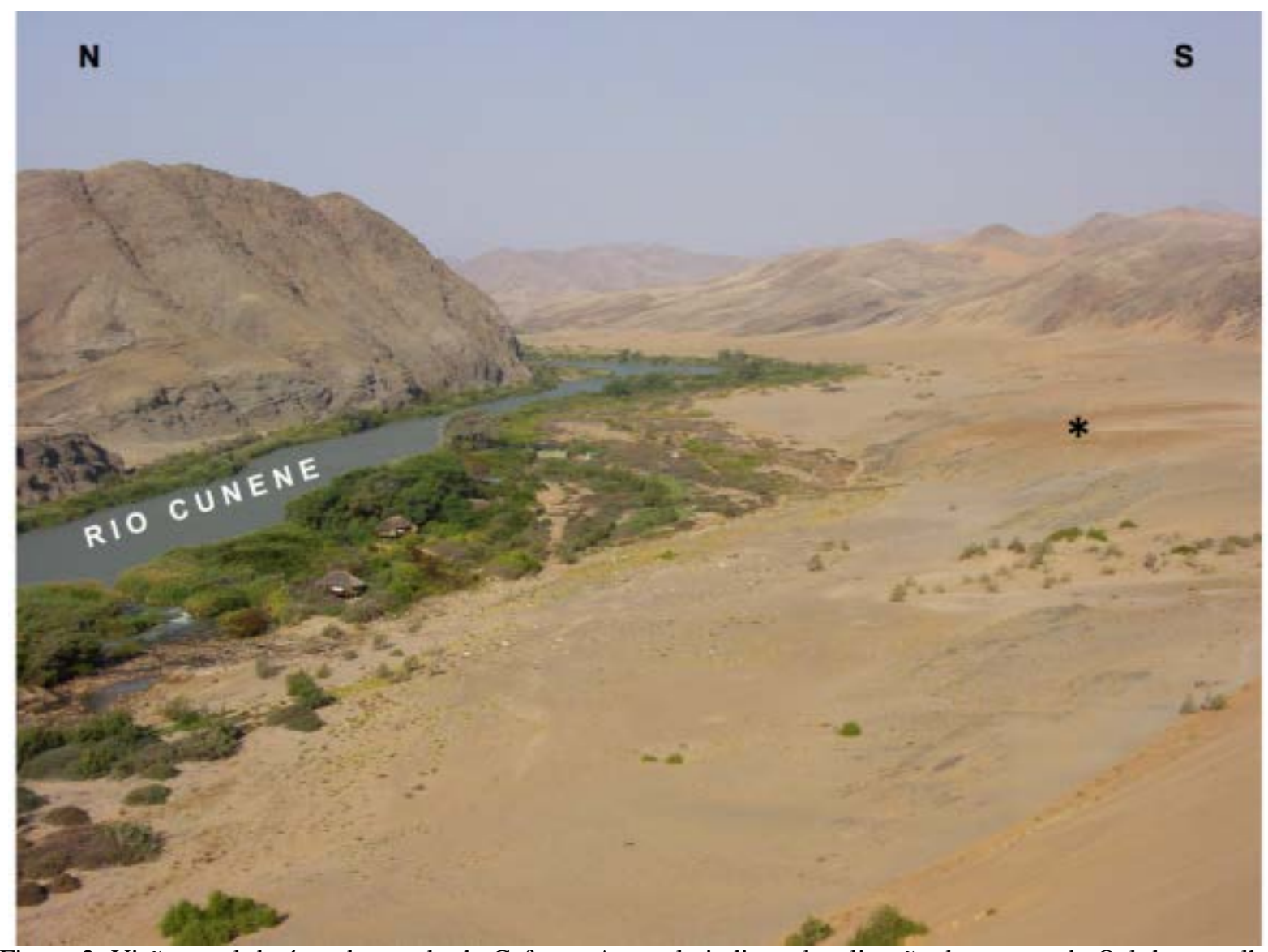

Figura 2. Visão geral da área de estudo de Cafema. A estrela indica a localização do terraço de Qal de cascalho de calhaus de quartzito ao longo da margem direita do Rio Cunene. A prospecção revelou a presença de 39 artefatos do PMA na superfície dentro da área de cascalhos no terraço do Rio Cunene.

Figure 2. Overview of Cafema study area. Star denotes location of the relict quartzite cobble-gravel Qal terrace along the left bank of the Cunene River. Survey revealed the presence of 39 MSA artifacts at the surface, within the cobble-gravel lag. 


\section{Materiais e métodos}

Durante uma prospecção regional em 2004-2007, a paisagem variada ao longo da fronteira entre Angola e Namíbia foi mapeada e descrita próxima à Serra Cafema, no lado namíbio da porção baixa do Rio Cunene. Métodos de levantamento padrão foram empregados, e características geoarqueológicas e vestígios culturais foram inventariados. Após a descoberta de artefatos a céu aberto no contexto do terraço, os vestígios do PMA foram macroscopicamente examinados (Tabela 1), e seus atributos petrográficos registrados (Nicoll 2009; 2010). Observações de campo iniciais sugeriam que o terraço Qal foi coberto por materiais bem grosseiros (Figura 3) incluindo seixos e calhaus de quartzito que podem representar matérias-primas adequadas (Figura 3A, C) para lascamento (Figura 3B).

Tabela 1. Achados arqueológicos em Cafema, Namíbia-Angola (Nicoll 2010). “?”indica incerteza. Table 1. Archaeological finds in Cafema, Namibia-Angola (Nicoll 2010). ”?” indicates uncertainty.

\begin{tabular}{llc}
\hline Descrição do artefato & Contexto do achado & Contagem \\
\hline Lasca simples (PMA) & Cluster A & 4 \\
Lasca simples (PMA) & Cluster B & 3 \\
Lasca simples (PMA) & Cluster C & 4 \\
Lasca simples (PMA) & Cluster D & 3 \\
Lasca simples (PMA) e sem diagnóstico & Cluster E & 3 \\
Lasca sem diagnóstico & Cluster F & 4 \\
Lasca simples (PMA?) (Figuras 4, 5A) & Contexto Isolado & 5 \\
Lasca simples - sem diagnóstico & Contexto Isolado & 2 \\
Núcleo Levallois (Musteriense?) & Contexto Isolado & 1 \\
Lamina Levallois (?) (Musteriense?) & Contexto Isolado & 2 \\
Lascas Levallois (Musteriense?) & Contexto Isolado & 5 \\
Ponta Levallois (Musteriense?) & Contexto Isolado & 3 \\
\hline & Total de achados & 39 \\
\hline
\end{tabular}

Pesquisas de campo adicionais foram realizadas em 2011. Para caracterizar o tamanho e a composição sedimentar de pedras de terraço do rio Cunene onde os artefatos foram descobertos, foram utilizados métodos granulométricos, mais precisamente uma modificação do método 'Wolman Pebble Count' (Contagem de Seixos de Wolman - Leopold et al. 1964; Bevenger \& King 1995). Próximo ao local de artefatos de Cafema, foram plotados três grids de transects geocodificados na superfície do terraço fluvial do Qal. Cada grid utilizou quatro fitas de 20 metros orientados nas direções cardinais (Norte-Sul, Leste-Oeste). Foram colocadas outras fitas de 20 metros na orientação N-S, nos 50 centímetros de intervalo a partir da prospecção de origem. Caminhando Norte-Sul ao longo da fita, foram observados os materiais de superfície e registrados o tamanho e litologia das maiores rochas observadas a cada 50 centímetros $(\mathrm{cm})$, em intervalos de $50 \mathrm{~cm}$. Em seguida, a fita N-S foi movida para o leste até o próximo intervalo de $50 \mathrm{~cm}$ ao longo da grade, e observações foram repetidas, registro do tamanho e composição em intervalos de $50 \mathrm{~cm}$. Ao fim de cada transito, a fita foi movida e as observações foram repetidas ao longo da grade.

As frações granulométricas utilizadas foram classificadas em: A - Areia $(<2 \mathrm{~mm})$; GF Granulo Fino (2-24 mm); GG - Granulo Grosso (25-64 mm); C - Calhau (65-255 mm); e M Matacão (>255 mm). As litologias primárias registradas foram: anfibolito, anortosito, basalto, carbonato, diabásio, gabro, gneisse, granito, mármore, obsidiana, quartzito, riolito, xisto, rochas metamórficas indiferenciadas, desconhecidas e outras.

Além disso, durante o período 2011-2012, foram avaliados mapas geológicos disponíveis para identificar afloramentos rochosos ao longo do Rio Cunene onde as partículas do terraço 
de litologias diferentes podem ter sido originadas. Dentro da região de estudo, foram mapeadas as áreas fonte de afloramento dos clastos fluviais compondo a unidade do terraço Qal. Isto foi realizado para identificar e delinear potenciais áreas de origem dos líticos.
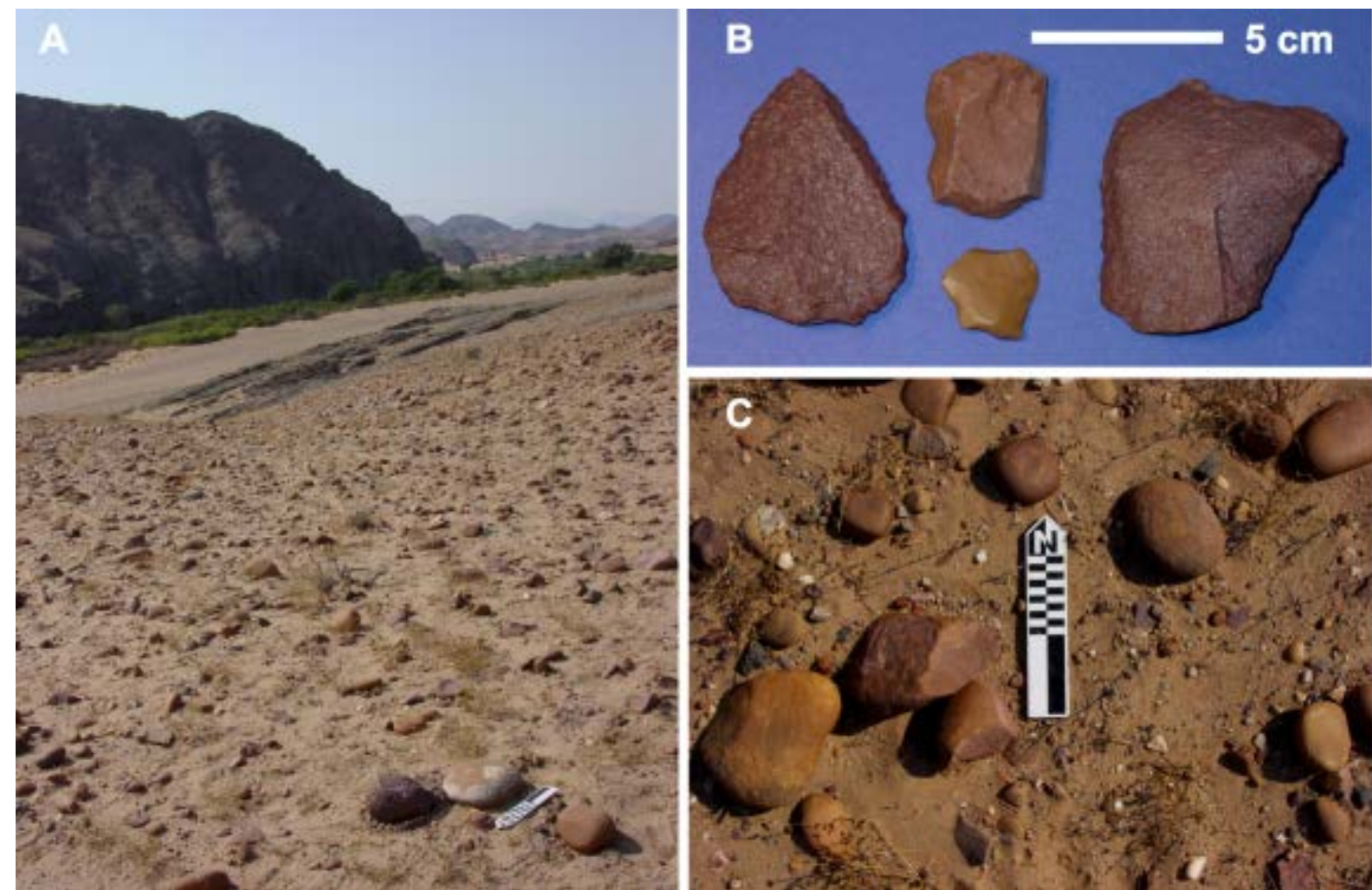

Figura 3. A) O terraço antigo fluvial. B) Artefatos descobertos. C) Imagem aproximada da superfície mostra Qal com clastos de quartzito bem arredondados, abrasados por vento, e patinados. Escala em centímetros.

Figure 3. A) The relict river terrace. B) Found artifacts. C) Close-up of the surface shows Qal with well-rounded, wind-abraded, patinated quartzite clasts. Scale in centimetres.

\section{Resultados}

Serão discutidas as áreas fonte em potencial para matérias primas líticas nesta região, e apresentada a hipótese de que o terraço Qal do Rio Cunene era uma fonte secundária de matéria-prima lítica durante o PMA. Serão discutidas também as potenciais áreas fonte de matérias primas nesta região.

Os resultados da contagem de seixos estão presentados na Tabela 2. Na área de prospecção, 2400 observações foram registradas. A contagem indica que o tamanho dos sedimentos da superfície no terraço é dominado por calhaus, que compõe $48 \%$ da contagem total. Cascalhos grosseiros somam $43 \%$ da contagem. Isso faz sentido, porque os artefatos do PMA foram recuperados em um terraço de cascalho.

A composição dos clastos do terraço é quase exclusivamente de rochas metamórficas (Figura 4). A litologia dominante registrada é o quartzito (80\%), seguida de gneisse ( 5\%). Rochas ultramáficas (Figura 4C), mármores, gabros, xistos e rochas vulcânicas foram representados em pequenas quantidades, e não havia obsidianas presentes no terraço. Os calhaus de quartzito preservados no terraço Qal são muito bem arredondados no geral, com marcas de percussão, e uma patina superficial de polimento arenoso. Alguns dos clastos de quartzito foram submetidos à esfoliação (Figura 4A) e lascamento a partir de intemperismo de sal (Figura 4D). Mais de 75\% dos artefatos do PMA recuperados da superfície do terraço são de composição quartzítica (Figura 4B). 
Tabela 2. Os resultados da contagem de seixos e a composição dos clastos.

Table 2. Results of pebble counts and clast composition.

\begin{tabular}{lccccc}
\hline Grade & $\mathbf{1}$ & $\mathbf{2}$ & $\mathbf{3}$ & Totais & Percentagem \\
\hline Tamanho (m) & $10 \times 10$ & $10 \times 10$ & $20 \times 20$ & & \\
Contagens Totais & 400 & 400 & 1600 & 2400 & \\
\hline Tamanho do Sedimento & & & & & \\
\hline S - Sand / Areia (<2 mm) & 2 & 0 & 26 & 28 & 1.17 \\
FG - Fine Gravel / Cascalho Fino (2-24 mm) & 14 & 9 & 126 & 149 & 6.21 \\
CG - Coarse Gravel / Cascalho Grosso (25-64 & 98 & 154 & 784 & 1036 & 43.17 \\
$\quad$ mm) & & & & & \\
CO - Cobble / Pedras (65-255 mm) & 283 & 231 & 638 & 1152 & 48.00 \\
BO - Boulder / Pedregulho (>255 mm) & 3 & 6 & 26 & 35 & 1.46 \\
\hline Composição da Partícula & & & & & \\
\hline anfibolito & 0 & 0 & 2 & 2 & 0.08 \\
anortosito & 3 & 1 & 7 & 11 & 0.46 \\
basalto & 4 & 0 & 3 & 7 & 0.29 \\
carbonato & 0 & 0 & 3 & 3 & 0.13 \\
dolerito & 2 & 1 & 2 & 5 & 0.21 \\
gabro & 7 & 0 & 4 & 11 & 0.46 \\
gneisse & 49 & 13 & 65 & 127 & 5.29 \\
granito & 8 & 2 & 21 & 31 & 1.29 \\
mármore & 0 & 0 & 1 & 1 & 0.04 \\
obsidiana & 0 & 0 & 0 & 0 & 0.00 \\
quartzito & 247 & 347 & 1326 & 1920 & 80.00 \\
riólito & 3 & 0 & 6 & 9 & 0.38 \\
xisto & 2 & 0 & 7 & 9 & 0.38 \\
metamórfica indiferenciado & 29 & 1 & 65 & 95 & 3.96 \\
desconhecido & 27 & 26 & 54 & 107 & 4.46 \\
outros & 19 & 9 & 34 & 62 & 2.58 \\
\hline
\end{tabular}

\section{Discussão e conclusões e considerações finais}

Com base na composição dos artefatos, está claro que a rocha silicosa era uma fonte importante para produção lítica durante o PMA. Isto é refletido no fato de que o material predominante na produção de instrumentos líticos recuperados em Cafema era o quartzito. Quartzito é conhecido por ser um material preferido para produção de instrumentos de tamanho consistente, facilidade para lascamento, qualidade de forma e contorno, e persistência na retenção dos gumes (Kuman 1996).

Para definir áreas de aquisição regionais onde as pessoas poderiam ter acessado matérias-primas de quartzito em escala local (0-5 km), regional (6-20 km) e supra-regional (21-100 km), nós examinamos os afloramentos geológicos na região. Devido à sua localização remota, a geologia desta região do sul da África não é bem conhecida, e os mapas disponíveis estão em escalar grosseira. Afloramentos ao longo do rio Cunene incluem algumas das rochas mais antigas do mundo datando do Vaaliano há 1760 milhões de anos, e compreendem parte de uma Grande Província Ígnea (GPI) que se estende através do continente Africano. Os afloramentos do leito rochoso local próximos à Cafema incluem um complexo metamórfico de grau médio a alto, granitoides, e rocha nativa (Kröner et al. 2015) estas criaram o terreno montanhoso acidentado entalhados pelo Rio Cunene (Geological Survey of South Africa and South West Africa 1980). 

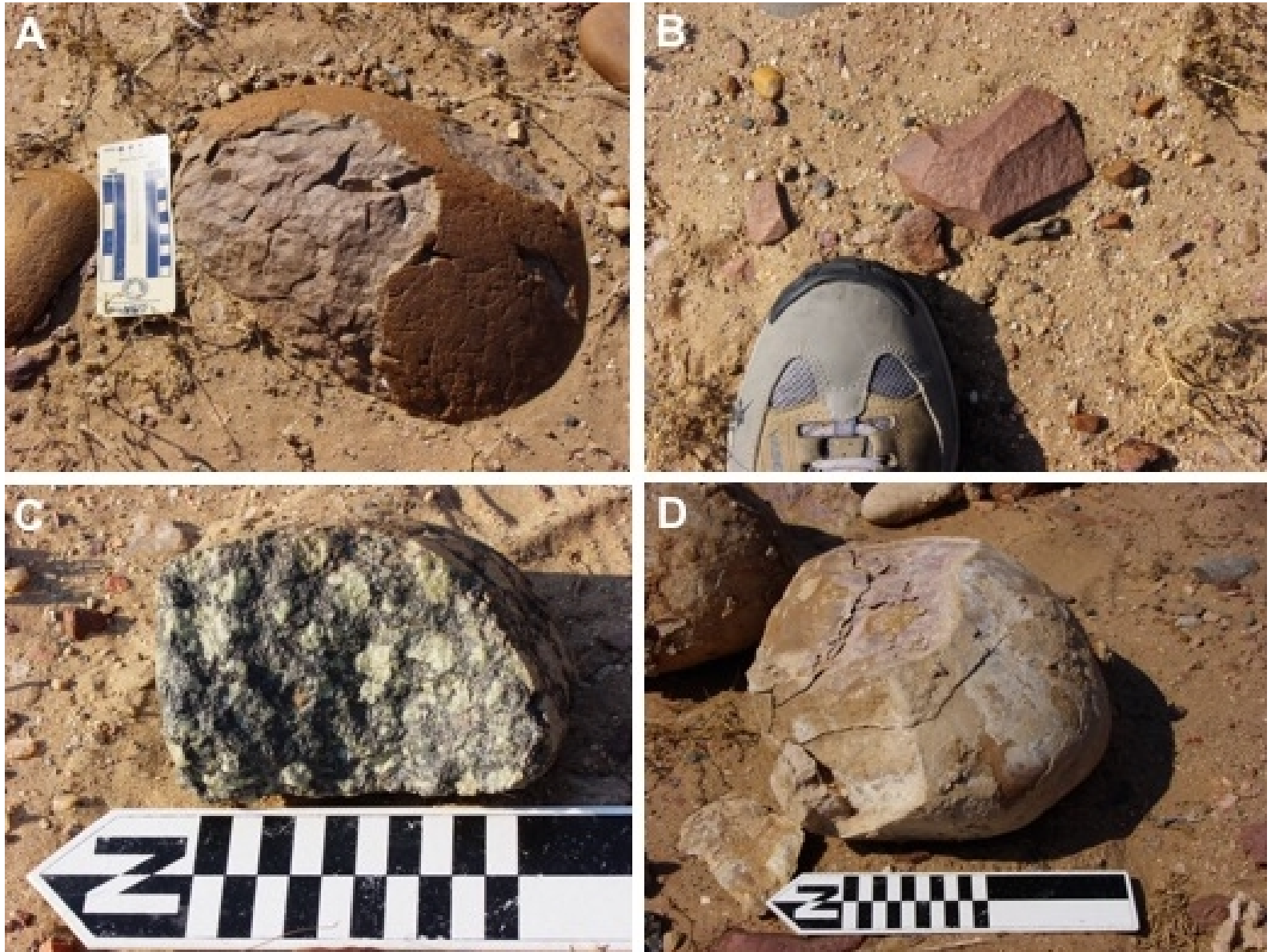

Figura 4. A) Matacão de quartzito esfoliado patinado. B) Achado de superfície: lítico de quartzito. C) Matacão ultramáfico (gabro) fraturado com cristais de olivina altamente intemperizados. D) Matacão de quartzito que passou por desbastamento devido a intemperismo termal e de sal. Escala em centímetros.

Figure 4. A) Patinated, exfoliated quartzite boulder. B) Surface find: quartzite lithic. C) Fractured ultramafic (gabbro) boulder with highly weathered olivine crystals. D) Quartzite boulder that has experienced spalling due to thermal and salt-weathering. Scale in centimetres.

A Tabela 3 lista as unidades geológicas primárias na área de estudo próximo à Cafema, sumarizado de acordo com a literatura publicada (Meier et al. 2008; 2013; Brandt \& Klemd 2008; Kröner et al. 2015, e referências citadas). Uma vez que quartzito é o tipo de clasto dominante ocorrendo como matérias-primas no terraço do rio onde os artefatos do PMA foram encontrados, nós podemos concluir que os próprios materiais do terraço do rio (Qal) estavam entre as prováveis áreas fonte de matéria prima usadas durante o Paleolítico Médio. Calhaus e matacões arredondados estão presentes como uma couraça superficial, junto com os artefatos no Qal dentro do terraço fluvial. O terraço fluvial em si é a fonte mais próxima dos artefatos recuperados.

Se a fonte de matéria-prima era aluvial (como na unidade de Qal), calhaus fluviais de quartzito podem ter derivado de afloramentos localizados a montante no sistema Cunene, que é uma rede fluvial maior (Figura 1) que drena uma diversidade de unidades geológicas. A localização exata das áreas fonte de quartzito primárias é impossível de definir ao nível de solo, uma vez que o terreno é inóspito e os afloramentos da área não são conhecidos em detalhe suficiente. Apesar de não ser possível identificar as áreas e formação precisas, nós podemos fazer algumas avaliações relativas sobre áreas fonte dentro da região, baseado na geologia. 
Tabela 3. Unidades geológicas primárias na área de estudo próximo Cafema. Ma = duração em milhões de anos. Table 3. Primary geological units in the studied area next to Cafema. $\mathrm{Ma}=$ millions of years.

\section{Idade}

geológica Abreviação Unidade Litologia

Eratema Cenozóica: Era Isotópica $1 \mathrm{Ma}$ ao dia moderno

\begin{tabular}{cll}
\hline$<1 \mathrm{Ma} \quad$ Qal & Quaternário & Modernos sedimentos superficiais incluem \\
& & dunas, sand sheets, depósitos fluviais e \\
& terraços, unidades lacustres e deltas, e \\
& minerais de evaporito.
\end{tabular}

\begin{tabular}{|c|c|c|c|}
\hline \multicolumn{4}{|c|}{ Eratema Namibiana/Cambriana: Era Isotópica 1000 Ma para 450 Ma } \\
\hline $\begin{array}{l}900-720 \\
\mathrm{Ma}\end{array}$ & Nda & $\begin{array}{l}\text { Damara } \\
\text { Seqüência }\end{array}$ & $\begin{array}{l}\text { Inclui as rochas arenosas do Grupo Nosib, isto } \\
\text { é, quartzitos, xistos e (meta)conglomerados. }\end{array}$ \\
\hline \multicolumn{4}{|c|}{ Eratema Mokoliana: Era Isotópica 2050 Ma para 1000 Ma } \\
\hline$\sim 1800 \mathrm{Ma}$ & $\mathrm{Mu}$ & [indiferenciado] & $\begin{array}{l}\text { Inclui a Formação Okapuka riólitos, basaltos, } \\
\text { andesitos, anfibolito, xisto verde e quartzito. }\end{array}$ \\
\hline $1363 \mathrm{Ma}$ & KIC & $\begin{array}{l}\text { Kunene Intrusivo } \\
\text { Complexo }\end{array}$ & $\begin{array}{l}\text { Ultramáficas. Anortosito, gabro, dunito, } \\
\text { forito, sienito, foyaite. intrusivo em Epupa } \\
\text { Complexo. Zonas de alteração e veios de } \\
\text { quartzo. }\end{array}$ \\
\hline
\end{tabular}

Eratema Vaaliana: Era Isotópica 2400 para $2050 \mathrm{Ma}$

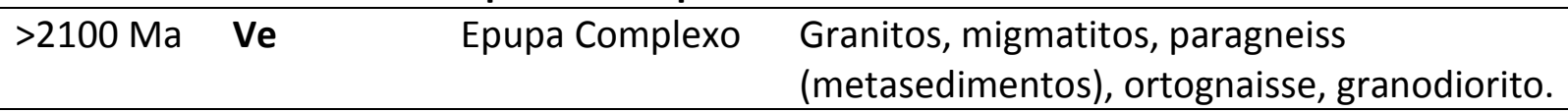

Muitas unidades geológicas podem ter contribuído potencialmente para os clastos presentes no terraço fluvial de Qal onde os artefatos do PMA foram descobertos. Os clastos de quartzito no Qal devem ter derivado da Sequência Damara (Nda) ou de litologias presentes dentro da indiferenciada unidade Mokoliana (Mu), os quis não são mapeados em detalhe. Clastos de granito são provavelmente originados do Complexo Epupa (Ve) e da formação Okapuka dentro das indiferenciadas unidades Mokolianas. Os gabros e clastos ultramáficos são derivados do Complexo Intrusivo Kunene (KIC).

As fontes primárias em potencial mais próximas (não aluvais) incluem quartzito nos afloramentos de Nda (tais como os não aluviais) estão localizados dentro de $2 \mathrm{~km}$ do sítio (Figura 5). No entanto, localizações de fontes (ou pedreiras) confirmadas de quartzito não têm sido identificadas especificamente em afloramentos. Clastos ultramáficos tais como os gabros e gneisses são provavelmente originários da unidade Ke, os quais afloram por cerca de 200 km acima no rio. Diferentemente de obsidiana, poucas técnicas analíticas explicitas existem para refinamento esta avaliação de proveniência de instrumentos de quartzito do PMA para uma escala local mais precisa (ex.: a obra clássica na África por Muir \& Hivernel 1976; Barut 1996; Negash \& Shackley 2006 e outros).

Para propor um modelo de aquisição plausível: o sistema do Rio Cunene transportou matérias-primas de quartzito do leito rochoso na base de drenagem, e depositou calhaus no terraço aluvial de Qal há 225 mil anos (datação em OSL-SAR em Nicoll 2010). Muitos calhaus de quartzito foram depositados como alúvio dentro do terraço de Qal, onde o quartzito era facilmente adquirido e núcleos eram lascados para produzir os instrumentos do PMA. Dispersões no sítio a céu aberto Cafema refletem as atividades simples e eficientes de caçadores-coletores móveis que exploravem a matérias-primas de terraços fluviais ou aluviais, pois estes eram fontes abundantes de matérias-primas preferidas. Esta reconstrução de áreas fonte da coleção de artefatos do PMA em Cafema enfatizam a importância dos corredores 
ribeirinhos para hominíneos durante e Pleistoceno Médio, o período marcado pela primeira aparição e dispersão da espécie humana moderna Homo sapiens.

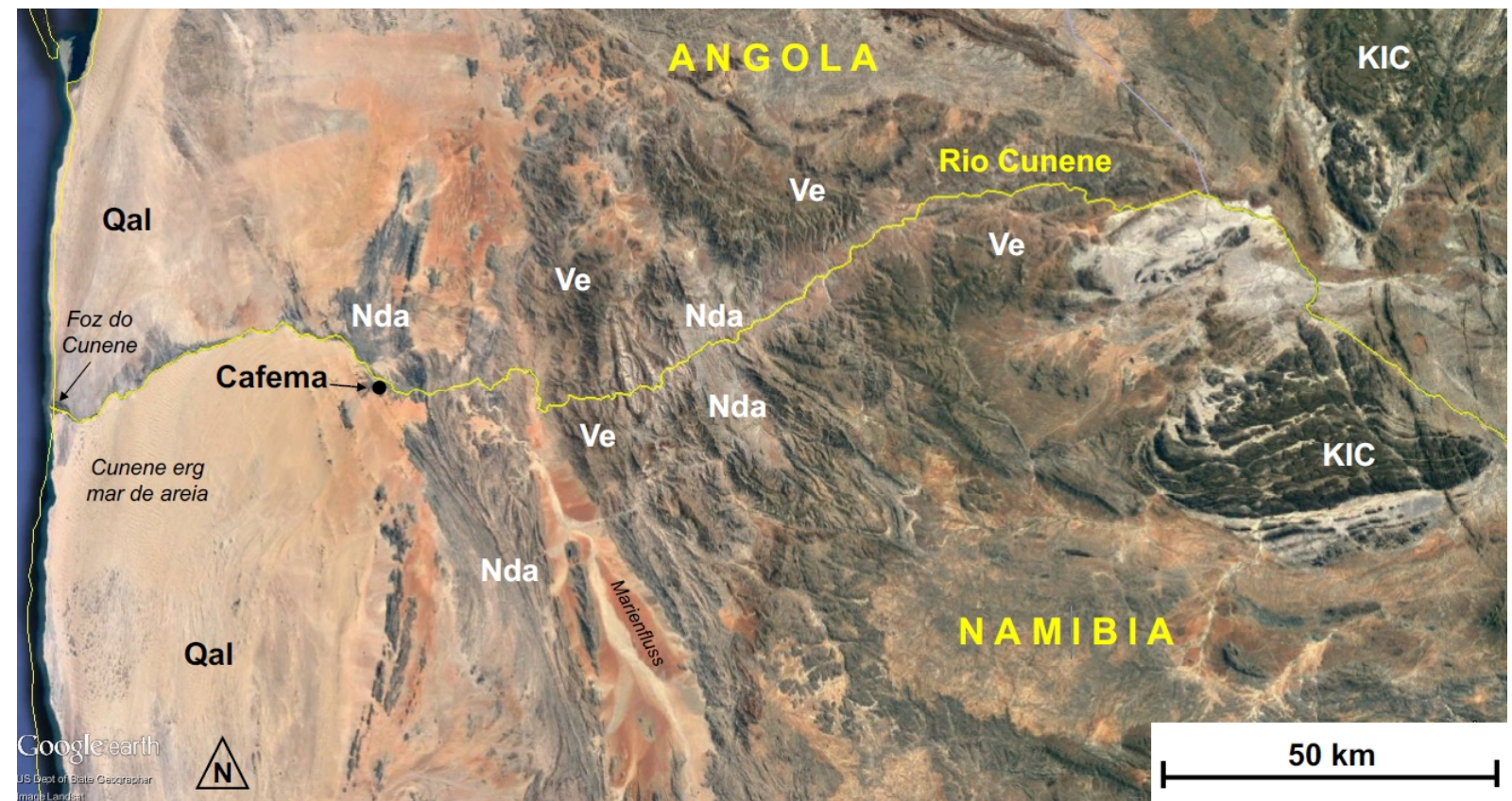

Figura 5. Mapa do sítio do Paleolítico Médio Africano (PMA) Cafema no Google Earth, ao longo do baixo Rio Cunene, fronteira entre Angola e Namíbia. As unidades geológicas de afloramento primário listada na Tabela 3 que contribuem com os clastos do terraço fluvial de Qal. KIC: Complexo Intrusivo Kunene. Ve: Complexo Epupa. Nda - Sequência Damara. Qal: Aluvium Quaternário.

Figure 5. Google Earth map of Cafema MSA site, along the Lower Cunene River, Angola-Namibia border. The primary outcropping geologic units listed in Table 3 that contribute clasts to the Qal in the relict river terrace. KIC: Kunene Intrusive Complex. Ve: Epupa Complex. Nda - Damara Sequence. Qal: Quaternary alluvium

\section{Agradecimentos}

À Royal Society, Royal Geographical Society e Brasenose College da University of Oxford pelo apoio financeiro do projeto. Ao Ministério do Ambiente e Turismo da Namíbia, por permitirem o estudo. À Wilderness Safaris por fornecer suporte logístico. Aos colegas arqueólogos africanistas, incluindo Nick Barton e Terry Hardaker. Graças e Richard Cote e Kevin Leishman por auxílio em campo. Mais importante ainda, agradeço a João Carlos Moreno de Sousa pela ajuda com a tradução para língua portuguesa. Agradeço também àqueles que revisaram este artigo; aprecio seu feedback útil para melhorar o artigo.

\section{Referencias}

Ambrose, S. H., \& Lorenz, K. G. 1990, Social and ecological models for the Middle Stone Age in southern Africa. In: The Emergence of Modern Humans, An archaeological perspective (Mellars, P., Ed.), Edinburgh University Press, Edinburgh: p. 3-33. (em inglês) ("Modelos sociais e ecológicos para o Paleolítico Médio no sul da Africa”)

Andrefsky, W. Jr. 1994, Raw Material Availability and the Organization of Technology. American Antiquity, 59: 21-34. (em inglês) ("Disponibilidade de Matéria Prima e a Origanização Tecnológica”) doi:10.2307/3085499

Andrefsky, W. Jr. 2005, Lithics: Macroscopic Approaches to Analysis $2^{\text {nd }}$ Edition. Cambridge University Press, Cambridge, 326 p. (em inglês) ("Líticos: Abordagens macroscópicas para análise”) 
Barut, S. 1996, Obsidian source use in the Later Stone Age at Lukenya Hill, Kenya. In: Aspects of African Archaeology (Pwiti, G., \& Soper, R., Eds.), University of Zimbabwe Publications, Harare: p. 297-306. (em inglês) ("Uso de fonte de obsidiana no Paleolítico Superior em Lukenya Hill, Quênia”)

Basell, L. S. 2008, Middle Stone Age (MSA) site distributions in eastern Africa and their relationship to Quaternary environmental change, refugia and the evolution of Homo sapiens. Quaternary Science Reviews, 27: 2484-2498. (em inglês) (“Distribuição de sítios do Paleolítico Médio no leste africano e suas relações com as mudanças ambientais no Quaternário, refúgios e a evolução do Homo sapiens”) doi:10.1016/j.quascirev.2008.09.010

Beck, C., \& Jones, G. T. 1990, Toolstone Selection and Lithic Technology in Early Great Basin Prehistory. Journal of Field Archaeology, 17: 283-299. (em inglês) ("Seleção de instrumentos de pedra e tecnologia lítica na pré-história da antiga grande bacia”) doi:10.1179/009346990791548240

Bevenger, G. S., \& King, R. M. 1995, A Pebble Count Procedure for Assessing Watershed Cumulative Effects. Res. Pap. RM-RP-319. U.S. Department of Agriculture, Forest Service, Rocky Mountain Forest and Range Experiment Station, Fort Collins, CO: p.110. (em inglês) ("Um procedimento de contagem de seixos para assessor os efeitos cumulativos de bacias fluviais”)

Binford, L. R. 1982, The archaeology of place. Journal of Anthropological Archaeology, 1: 531. (em inglês) (“A arqueologia do lugar”) doi:10.1016/0278-4165(82)90006-X

Brandt, S., \& Klemd, R. 2008, Upper-amphibolite facies partial melting of paragneisses from the Epupa Complex, NW Namibia, and relations to Mesoproterozoic anorthosite magmatism. Journal of Metamorphic Geology, 26: 871 - 893. (em inglês) ("Facies de anfibolito superior do derretimento parcial de paragneisses do complexo de Epupa, Noroeste da Namíbia, e relações com magmatismo de anorthosita mesoproterozóica”) doi:10.1111/j.1525-1314.2008.00793.x

Brantingham, P. J., \& Kuhn, S. L. 2001, Constraints on Levallois Core Technology: A Mathematical Model. Journal of Archaeological Science, 28: 747-761. (em inglês) ("Restrições da tecnologia do núcleo Levallois: Um modelo matemático") doi:10.1006/jasc.2000.0594

Brown K. S. et al. 2012, An early and enduring advanced technology originating 71000 years ago in South Africa. Nature, 491: 590-593. (em inglês) ("Uma antiga e duradoura tecnologia avançada originada 710.000 anos atrás na África do Sul”) doi:10.1038/nature11660

Clark, J. D. 1966, The distribution of prehistoric culture in Angola. Subsidios para a história, arqueologia et etnografia dos povos da Lunda Vol. 73, Companhia de Diamantes de Angola (Museu do Dundo), Lisboa, 102 p. (em inglês) ("A distribuição da cultura préhistórica em Angola”)

Clark, J. D. 1967, Atlas of African Prehistory. University of Chicago Press, Chicago, 62 p. (em inglês) (“Atlas da Pré-História Africana”)

Conard, N. J. 2010, Cultural modernity: Consensus or conundrum? Proceedings of the National Academy of Sciences of the United States of America, 107: 7621-7622. (em inglês) (“Modernidade cultural: Consenso ou enigma?”) doi:10.1073/pnas.1001458107 
Dibble, H. L., \& Bar-Yosef O. (Eds.) 1995, The Definition and Interpretation of Levallois Technology. Monographs in World Archaeology, Madison, 77 p. (em inglês) (“A definição e interpretação da tecnologia Levallois”)

Dusseldorp, G. L. 2011, Tracking the influence of technological change on Middle Stone Age hunting strategies in South Africa. Quaternary International, 270: 70-9.. (em inglês) ("Rastreando a influência da mudança tecnológica nas estratégias de caça durante o Paleolítico Médio na África do Sul”) doi:10.1016/j.quaint.2011.02. 011

d'Errico, F. 2003, The invisible frontier. A multiple species model for the origin of behavioural modernity. Evolutionary Anthropology, 12: 188-202. (em inglês) (“A fronteira invisível. Um modelo de espécies múltiplas para a origem da modernidade comportamental”)

d'Errico, F., \& Henshilwood, C. S. 2011, The origin of symbolically mediated behaviour: From antagonistic scenarios to a unified research strategy. In: Homo Symbolicus: The dawn of language, imagination and spirituality (Henshilwood, C. S., \& d'Errico, F., Eds.), John Benjamins, Amsterdam: p. 49-73. (em inglês) (“A origem do comportamento mediado simbolicamente: Dos cenários antagônicos para uma estratégia de pesquisa unificada")

d’Errico, F., Henshilwood, C. S., Lawson, G., Vanhaeren, M., Tillier, A., Soressi, M., Bresson, F., Maureille, B., Nowell, A., Lakarra, J., Backwell, L., \& Julien, M. 2003, Archaeological evidence for the emergence of language, symbolism, and music: An alternative multidisciplinary perspective. Journal of World Prehistory, 17: 1-70. (em inglês) ("Evidência arqueológica para a emergência da linguagem, simbolismo e música: Uma perspectiva multidisciplinar alternativa")

Geological Survey of South Africa and South West Africa 1980, South West Africa/Namibia: Geological Map, 1:1.000.000, Windhoek, Namibia. (em inglês) ("Mapa geológico do sudoeste africano: Namíbia”)

Henshilwood, C. S., d’Errico, F., Yates, R., Jacobs, Z., Tribolo, C., Duller, G. A. T., Mercier, N., Sealy, J. C., Valladas, H., Watts, I., \& Wintle, A. G. 2002, Emergence of modern human behaviour: Middle Stone Age engravings from South Africa. Science, 295: 1278-1280. (em inglês) ("Emergência do comportamento humano moderno: Gravuras do Paleolítico Médio da África do Sul”) doi:10.1126/science.1067575

Henshilwood, C. S., \& Marean, C. W. 2003, The origin of modern human behaviour: A review and critique of models and test implications. Current Anthropology, 44: 627651. (em inglês) ("A origem do comportamento humano: Uma revisão e crítica das implicações de modelos e testes”) doi:10.1086/377665

Henshilwood, C. S., \& Marean, C. E. 2006, Remodeling the origins of modern human behaviour: Tracing the lineage of modern man. In: The prehistory of Africa: Tracing the lineage of modern man (Soodyall, H., Ed.), Jonathan Ball, Cape Town: p. 37-59. (em inglês) ("Remodelando as origens do comportamento humano: Traçando a linhagem do humanos modernos")

Henshilwood, C. S., \& Dubreuil, B. 2011, The Still Bay and Howiesons Poort, 77-59 ka: Symbolic material culture and the evolution of the mind during the African Middle Stone Age. Current Anthropology, 52: 361-400. (em inglês) ("Still Bay e Howiesons Poort, 77-59 mil AP: Cultura material e a evolução da mentedurante o Paleolítico Médio africano”) doi:10.1086/660022 
Henshilwood, C. S., \& d'Errico, F. 2011, Middle Stone Age engravings and their significance for the debate on the emergence of symbolic material cultures. In: Homo Symbolicus: The dawn of language, imagination and spirituality (Henshilwood, C. S., \& d'Errico, F., Eds.), John Benjamins, Amsterdam: p. 75-96. (em inglês) (“Gravuras do Paleolítico Médio e sua significância para o debate na emergência das culturas materiais simbólicas”)

Jacobs, Z., Roberts, R. G., Galbraith, R. F., Deacon, H. J., Grün, R., Mackay, A., Mitchell, P., Vogelsang, R., \& Wadley, L. 2008, Ages for the Middle Stone Age of Southern Africa: Implications for human behavior and dispersal. Science, 322: 733-5. (em inglês) ("Idades para o Paleolítico Médio no sudeste africano: Implicações para o comportamento e a dispersão humana”) doi:10.1126/science.1162219

Klein, R. G. 1995, Anatomy, Behavior, and Modern Human Origins. Journal of World Prehistory, 9: 167-198. (em inglês) ("Origens da anatomia e comportamento de humanos modernos”) doi:10.1007/BF02221838

Klein, R. G. 1999. The Human Career. University of Chicago Press, Chicago, 1024 p. (em inglês) (“A carreira humana”)

Kröner, A., Rojas-Agramonte, Y., Wong, J., \& Wilde, S. A. 2015, Zircon reconnaissance dating of Proterozoic gneisses along the Kunene River of northwestern Namibia. Tectonophysics, 662: 125-139. (em inglês) ("Reconhecimento de datações de zircão de gnaisses proterozóicos ao longo do rio Kunene, Noroeste da Namíbia”) doi:10.1016/j.tecto.2015.04.020

Kuman, K. 1996, The Oldowan Industry from Sterkfontein: raw materials and core forms. In: Aspects of African Archaeology (Pwiti, G., \& Soper, R., Eds), University of Zimbabwe, Harare: p. 139-146. (em inglês) (“A indústria Olduvaiense de Sterkfontein: formas de matérias-primas e núcleos”)

Kyara, O. 1996, Lithic raw materials as an epistemological tool for hominid behavioral reconstruction: A preliminary overview, In: Aspects of African Archaeology. (Pwiti, G., \& Soper, R., Eds.), University of Zimbabwe Publications, Harare: p. 111-120. (em inglês) ("Matérias primas líticas como um instrumento epistemológico para a reconstrução do comportamento hominíneo")

Leopold, L. B., Wolman, M., \& Miller, J. 1964, Fluvial Processes in Geomorphology. W. H. Freeman, San Francisco, 544 p. (em inglês) ("Processos fluviais em geomorfologia”)

Lycett, S. J., \& Eren, M. I. 2013, Levallois economics: An examination of 'waste' production in experimental produced Levallois reduction sequences, Journal of Archaeological Science, 40: 2384-2392. (em inglês) ("Economia Levallois: Uma análise de produção de "lixo” em sequencias de redução experimental de produção Levallois”) doi:10.1016/j.jas.2013.01.016

Lycett, S. J., \& von Cramon-Taubadel, N. 2013, A 3D morphometric analysis of surface geometry in Levallois cores: Patterns of stability and variability across regions and their implications. Journal of Archaeological Science, 40: 1508-1517. (em inglês) ("Uma análise morfométricas 3D da superfície geométrica em núcleos Levallois: Padrões de estabilidade e variabilidade através de regiões e suas implicações”) doi:10.1016/j.jas.2012.11.005

Maier, W. D., Teigler, B., \& Miller, R. 2008, The Kunene anorthosite complex and its satellite intrusions. In: The Geology of Namibia (Miller, R. Mc.G., Ed.), Geological 
Survey of Namibia, Windhoek: 9-1 to 9-18. (em inglês) (“O complexo de anortosita do Kunene e suas intrusões satélites”)

Maier, W. D., Rasmussen, B., Fletcher, I. R., Li, C., Barnes, S-J., \& Huhma, H. 2013, The Kunene Anorthosite Complex, Namibia, and Its Satellite Intrusions: Geochemistry, Geochronology, and Economic Potential. Economic Geology, 108: 953-986. (em inglês) ("O complexo de anartosita do Kunene, Namíbia, e suas intrusões satélites: Geoquímica, geocronologia e potencial econômico”) doi:10.2113/econgeo.108.5.953

McBrearty, S., \& Brooks, A. S. 2000, The revolution that wasn't: A new interpretation of the origin of modern human behavior. Journal of Human Evolution, 39:453-563. (em inglês) ("A revolução que não foi: Uma nova interpretação da origem do comportamento humano moderno”) doi:10.1006/jhev.2000.0435

McCoy, M. D., \& Ladefoged, T. N. 2009, New developments in the use of spatial technology in archaeology. Journal of Archaeological Research, 17: 263-295. (em inglês) ("Novos desenvolvimentos no uso de tecnologia espacial em arqueologia”) doi:10.1007/s10814009-9030-1

Mellars, P. 2006, Why did modern human populations disperse from Africa ca. 60,000 years ago? A new model. Proceedings of the National Academy of Sciences of the United States of America, 103(25): 9381-9386. (em inglês) ("Por que populações humanas modernas dispersaram da África 60 mil anos atrás? Um novo modelo”) doi:10.1073/pnas.0510792103

Mitchell, P. 2002, The Archaeology of Southern Africa. Cambridge University Press, Cambridge, 532 p. (em inglês) (“A arqueologia do sul africano”)

Muir, I. D., \& Hivernel, F. 1976, Obsidians from the Melka-Kunture Prehistoric Site, Ethiopia. Journal of Archaeological Science, 3: 211-217. (em inglês) (“Obsidianas do sítio pré-hitórico Melka-Konture, Etiópia”)

Negash, A., \& Shackley, M. S. 2006, Geochemical provenance of obsidian artifacts from the MSA site of Porc Epic, Ethiopia. Archaeometry, 48(1): 1-12. (em inglês) ("Proveniência geoquímica de artefato de obsidiana do sítio do Paleolítico Médio Porc Epic, Etiópia”) doi:10.1111/j.1475-4754.2006.00239.x

Newman, J. R. 1994, The Effects of Distance on Lithic Material Reduction Technology. Journal of Field Archaeology, 21: 491-501. (em inglês) ("Os efeitos da distância na tecnologia de redução de materiais líticos”) doi:10.1179/009346994797175541

Nicoll, K. 2009, Evidence of Levallois Technique at a Middle Stone Age open air site along the Angola-Namibia border. Antiquity, 83(319). (em inglês) ("Evidência da técnica Levallois em um sítio a céu aberto do Paleolítico Médio na fronteira entre Angola e Namíbia”) URL: http://antiquity.ac.uk/projgall/nicoll/

Nicoll, K. 2010, Geomorphic development and Middle Stone Age archaeology of the Lower Cunene River, Namibia-Angola Border. Quaternary Science Reviews, 29: 1419-1431. (em inglês) ("Desenvolvimento geomórfico e arqueologia do Paleolítico Médio no baixo rio Cunene, fronteira entre Namíbia e Angola”) doi:10.1016/j.quascirev.2009.02.026

Oestmo, S., Schoville, B. J., Wilkins, J., \& Marean, C. W. 2014, A Middle Stone Age Paleoscape near the Pinnacle Point caves, Vleesbaai, South Africa. Quaternary International, 350: 147-68. (em inglês) ("Uma paleopaisagem do Paleolítico Médio 
próximo às cavernas de Pinnaple Point, Vleesbaai, África do Sul”) doi:10.1016/j.quaint.2014.07.043

de la Peña, P. 2015, Refining Our Understanding of Howiesons Poort Lithic Technology: The Evidence from Grey Rocky Layer in Sibudu Cave (KwaZulu-Natal, South Africa). PLoS ONE, 10: e0143451. (em inglês) ("Refinando nosso entendimento da tecnologia lítica de Howiesons Poort: A evidência de camada rochosa cinza em Sibudu Cave, KwaZulu-Natal, África do Sul”) doi:10.1371/journal.pone.0143451

Porraz, G., Parkington, J. E., Rigaud, J.-P., Miller, C. E., Poggenpoel, C., Tribolo, C., Archer, W., Cartwright, C. R., Charrié-Duhaut, A., Dayet, L., Igreja, M., Mercier, N., Schmidt, P., Verna, C., \& Texier, P-J. 2013, The MSA sequence of Diepkloof and the history of southern African Late Pleistocene populations. Journal of Archaeological Science, 40: 3542-3552. (em inglês) (“A sequência do Paleolítico Médio de Diepkloof e a história das populações no Pleistoceno final no sul africano”) doi:10.1016/j.jas.2013.02.012

Renfrew, C., Dixon, J. E., \& Cann, J. R. 1969, Further Analysis of Near East Obsidian. Proceedings of the Prehistoric Society, 34: 319-331. (em inglês) (“Análises adicionais da obsidiana do oriente próximo”) doi:10.1017/S0079497X000139

Renfrew, C. 1972, The Emergence of Civilisation -The Cyclades and the Aegean in the third Millennium BC. Methuen Publishing Ltd., London, 627 p. (em inglês) (“A emergência da civilização - O Cyclades e o Aegean no terceiro milênio BC”)

Rogers, M. J. 1996, Lithic analysis in landscape archaeology: Evidence from East Turkana. In: Aspects of African Archaeology. (Pwiti, G., \& Soper, R., Eds.), University of Zimbabwe Publications, Harare: p. 103-109. (em inglês) (“Análise lítica em arqueologia da paisagem: Evidência do leste de Turkana”)

De Ruiter, D. J., Brophy, J. K., Lewis, P. J., Churchill, S. E., \& Berger, L. R. 2008, Faunal assemblage composition and paleoenvironment of Plovers Lake, a Middle Stone Age locality in Gauteng Province, South Africa. Journal of Human Evolution, 55: 1102-17. (em inglês) ("Composição de coleções faunísticas e paleoambiente de Plovers Lake, um sítio do Paleolítico Médio na província de Gauteng, África do Sul”) doi:10.1016/j.jhevol.2008.07.011

Shultz, S., Nelson, E., \& Dunbar, R. I. M. 2012, Hominin cognitive evolution: Identifying patterns and processes in the fossil and archaeological record. Philosophical Transactions of the Royal Society B, 367: 2130-2140. (em inglês) ("Evolução cognitiva hominínea: Identificando padrões e processos no registro fóssil e arqueológico”) doi:10.1098/rstb.2012.0115

Vogelsang, R. 1998, Archaeological survey in Kaokoveld, Namibia. Nyame Akuma, 50: 2224. (em inglês) ("Levantamento arqueológico em Kaokoveld, Namíbia”)

Wadley L. 2015, Those marvellous millennia: The Middle Stone Age of Southern Africa. Azania, 50(2): 155-226. (em inglês) (“Aqueles milênios maravilhosos: O Paleolítico Médio do sul africano")

Will, M., Bader, G. D., \& Conard, N. J. 2014, Characterizing the Late Pleistocene MSA Lithic Technology of Sibudu, KwaZulu-Natal, South Africa. PLoS ONE, 9(5): e98359. (em inglês) ("Caracterizando a tecnologia do Paleolítico Médio durante Pleistoceno Final em Sibudu, KwaZulu-Natal, África do Sul”) doi:10.1371/journal.pone.0098359 


\title{
Sourcing river rock and Middle Stone Age artifacts discovered along the Cunene River, Angola-Namibia border
}

\author{
Kathleen Nicoll \\ University of Utah, Department of Geography, Salt Lake City, Utah, U.S.A. Email: kathleen.nicoll@gmail.com
}

\begin{abstract}
:
Although many important prehistoric sites are known from South Africa, few comparable contexts have been discovered and documented in the northern Namibia and southern Angola borderlands. During a geomorphic assessment of riparian corridors in Namibia's Kaokoveld region, Middle Stone Age (MSA in Africa; broadly correlative with European Paleolithic) lithic artifacts were found preserved in unstratified plein air sites located atop a terrace adjacent to the perennial Cunene River. These remains attest to hominin activities along the northern edge of the modern hyperarid Namib Desert, which receives less than $100 \mathrm{~mm}$ of rainfall in a year. The location of the archaeological site is quite remote, and is along the eastern perimeter of the hyperarid Cunene erg (sandsea), and downstream of the Marienfluss-Hartmann Valley near Serra Cafema, At the Cafema site (as it is known), more than 30 lithic artifacts are preserved in the Cunene River valley, in context of a former river terrace. The artifacts discovered by a walking survey include quartzite flakes, cores, and points with some edge abrasion and varnish, including the first Levallois-Mousterian points found in this region of Africa. Since the archaeology of this area is poorly known, these cultural assemblages enable initial correlations across the continent, and provide a basis for reconstructing provenience, procurement and tool manufacture during the Middle Pleistocene, the time frame marked by the first appearance and the dispersal of the modern human species Homo sapiens.

To assess the potential source areas for lithic raw materials in this region, pebble counting methods and compositional assessment were conducted on the artifacts and Quaternary alluvium (Qal) of the relict river terrace at Cafema. Based on the composition of the artifacts found on the surface, siliceous rock was an important resource for lithic manufacture during the MSA; this is reflected in the observation that quartzite was overwhelmingly the dominant material used in the stone tool manufacture. Quartzite is generally known to be a preferred material for making tools of consistent size, ease of knapping, quality of form-shape, and persistence of edge retention.

To define regional procurement areas where hominin may have acquired quartzite raw materials at local $(0-5 \mathrm{~km})$, regional $(6-20 \mathrm{~km})$ and supra-regional $(21-100 \mathrm{~km})$ scales, we examined the geological outcrops in the region to identify potential sources. Due to its remoteness, the geology of this region of southern Africa is not well known, and the available maps are only available at coarse scales of resolution. Geologic outcrops along the Cunene River include some of the world's oldest rocks dating to the Vaalian $\sim 1760 \mathrm{Ma}$, and comprise part of a Large Igneous Province (LIP) that stretches across the African continent. The local bedrock outcrops near Cafema include a medium-tohigh grade metamorphic complex, granitoids, and surrounding country rock -- these rocks comprise the rugged mountainous terrain incised by the Cunene River.

Since quartzite is the dominant clast type occurring as raw material in the river terrace where the MSA artifacts themselves were found, we can conclude that river terrace materials (Qal) themselves were among the likely raw material sources exploited during antiquity. Rounded quartzite boulders and cobbles are present as surface lag, along with the artifacts in the Qal within the relict river terrace
\end{abstract}


at the plein air site. The river terrace itself is the closest source to the observed artifacts. If the raw material source was alluvial (i.e., within the Qal unit), quartzite river cobbles may have been derived from outcrops located further upstream the Cunene system, which is a large river network that drains a diversity of geologic units. Although it is not possible to identify the precise formation and procurement area of origin, we offer some relative assessments about likely source areas within the region, based on the geology. The specific geologic units that may have contributed quartzite clasts to the Qal river terrace include the (1) Damara Sequence (Nda); and (2) lithologies within the undifferentiated Mokolian unit, which are not well mapped in detail. The closest potential primary sources of quartzite raw materials in Nda rock outcrops (i.e., not alluvium within the Qal terrace at the site) are located within $2 \mathrm{~km}$ of the Cafema site. However, confirmed source locales in the past could not be specifically identified in the field.

The hypothesis offered is that the Qal alluvial components within the relict terrace of the Cunene River was a preferred source for quartzite lithic raw materials used by mobile hunter-gatherers to make tools during the MSA, sometime after 225 kya. Cafema is the first MSA site in northern Namibia that is in direct stratigraphic context with a securely dated unit. A replicate OSL-SAR date $\sim 220 \mathrm{kyr}$ has provided initial age constraints on a sandy unit preserved within the cobble-boulder Qal terrace fill, and constrains the maximum age for the overlying archaeological assemblage. These findings advance the reconstruction of this cultural landscape through a geoarchaeological lens, and form a basis for understanding the relict Pleistocene landscape and environment, its plant resources, and proximity to raw material sources within the riparian corridor of the perennial Cunene River.

Keywords: African Middle Stone Age; quartzite; lithic source areas; Quaternary alluvium (Qal); Levallois; provenance reconstruction 